\title{
Operators Characterized by Certain Cauchy-Schwarz Type Inequalities
}

By

\author{
Hideharu WATANABE*
}

\begin{abstract}
A Hilbert space operator $T$ satisfying

either (**) $|(T \xi, \eta)|^{2} \leqq(|T| \xi, \xi)\left(|T| \gamma_{1}, \eta\right)$ for all $\xi, \eta \in \mathcal{H}$, or $\quad(*) \quad|(T \bar{\xi}, \xi)| \leqq(|T| \xi, \xi)$ for all $\xi \in \mathscr{H}$

is studied. The condition (**) defines a slightly larger class than the hyponormality, and for compact operators $(* *)$ is equivalent to the normality. The condition $(*)$ is characterized by using an operator whose numerical radius is less than 1 , and among other things we show that $(*)$ and the normality are equivalent for matrices. Moreover, we show that $(*)$ and the normality are equivalent for trace class operators in Appendix.
\end{abstract}

\section{§. Introduction}

The purpose of the present paper is to study operators $T$ satisfying either $(* *)$

$$
|(T \xi, \eta)|^{2} \leqq(|T| \xi, \xi)(|T| \eta, \eta) \quad \text { for all } \xi, \eta \in \mathscr{H},
$$

or

$$
|(T \xi, \xi)| \leqq(|T| \xi, \xi) \quad \text { for all } \xi \in \mathscr{H} .
$$

Here, $T$ is an operator on a Hilbert space $\mathscr{H}$ with absolute value $|T|=\left(T^{*} T\right)^{1 / 2}$.

It is obvious that $(* *)$ implies (*). Based on the Cauchy-Schwarz inequality, one can show that $(* *)$ is equivalent to the operator inequality $\left|T^{*}\right| \leqq|T|$ (Theorem 1.1). In particular, if $T$ is normal (i. e., $T T^{*}=T^{*} T$ ), then (**) holds. In the operator theory, several extensions of the notion of the normality are known (see, for example, [8]). One of the most important and most widely studied classes among them is the hyponormality (i.e., $T T^{*} \leqq T^{*} T$ ) (see, for example, [5]). Since the square root function $t^{1 / 2}(t \geqq 0)$ preserves the (natural) order among positive operators ([7]), a hyponormal operator $T$ actually satisfies $\left|T^{*}\right| \leqq|T|$ (and hence (**)). Therefore, we are looking at a slightly (and strictly $\cdots$ see the end of $\S 1$ ) larger class than the hyponormality.

Communicated by H. Araki, April 22, 1993.

1991 Mathematics Subject Classifications : 47B20.

* Department of Mathematics, Faculty of Science, Kyushu University, Fukuoka 812, Japan. 
In $\S 1$, we will identify the class of operators satisfying (**). We will also show that for compact operators the validity of $(* *)$ is equivalent to the normality based on the following result due to $\mathrm{T}$. Ando ([1]): A compact hyponormal operator is automatically normal (see also [4] and [9]).

In $\S 2$, we will consider the condition $(*)$. Firstly we will characterize $(*)$ by making use of an operator $X$ whose numerical radius $w(X)$ satisfies $w(X) \leqq 1$ (Theorem 2.1). Secondly we will also show that for (finite) matrices $(\operatorname{dim}(\mathscr{H})$ $<\infty)$ the condition $(* *)$ actually implies the normality (Theorem 2.3). Consequently, $(*),(* *)$, and the normality are all equivalent for matrices.

A beautiful characterization of an operator $X$ with $w(X) \leqq 1$ was obtained by $T$. Ando ([2]). Based on this characterization and Theorem 2.1, in $\S 3$ we will show that the class of operators satisfying $(*)$ is strictly larger than the class of operators satisfying $(* *)($ when $\operatorname{dim}(\mathscr{H})=\infty)$.

Finally, in Appendix, we will extend to the result obtained in $\S 2$ to trace class operators. Based on T. Ando's factorization of a numerical contraction $X$ (i.e., $w(X) \leqq 1)([2])$, we will show that a numerical contraction and its adjoint have the same invariant vectors, and that for trace class operators the validity of $(*)$ is equivalent to the normality.

The results in Appendix were suggested by the referee, and the author would like to thank the referee for the suggestion.

\section{§1. Inequality $(* *)$}

In this section, we consider the following inequality for an operator $T \in$ $\mathscr{B}(\mathscr{H})$ :

$$
|(T \xi, \eta)|^{2} \leqq(|T| \xi, \xi)(|T| \eta, \eta) \quad \text { for all } \xi, \eta \in \mathscr{H}
$$

Theorem 1.1. For an operator $T \in \mathscr{B}(\mathscr{H}),(* *)$ holds for all $\xi, \eta \in \mathscr{H}$ if and only if $\left|T^{*}\right| \leqq|T|$.

Proof. Let $T=U|T|$ be the polar decomposition of $T$. Then, since $\left|T^{*}\right|$ $=U|T| U^{*}$,

$$
\begin{aligned}
|(T \xi, \eta)|^{2} & =\left|\left(U|T|^{1 / 2}|T|^{1 / 2} \xi, \eta\right)\right|^{2} \\
& =\left|\left(|T|^{1 / 2} \xi,|T|^{1 / 2} U^{*} \eta\right)\right|^{2} \\
& \leqq\left\||T|^{1 / 2} \xi\right\|^{2} \cdot\left\||T|^{1 / 2} U^{*} \eta\right\|^{2} \\
& =(|T| \xi, \xi)\left(U|T| U^{*} \eta, \eta\right) \\
& =(|T| \xi, \xi)\left(\left|T^{*}\right| \eta, \eta\right)
\end{aligned}
$$

for all $\xi, \eta \in \mathscr{H}$. Therefore, if $\left|T^{*}\right| \leqq|T|$, then we get (**).

Conversely when $(* *)$ is valid, by replacing $\xi, \eta$ by $U^{*} \xi$, $\xi$, we get 


$$
\begin{aligned}
\left|\left(\left|T^{*}\right| \xi, \xi\right)\right|^{2} & =\left|\left(U|T| U^{* \xi}, \xi\right)\right|^{2} \\
& =\left|\left(T U^{* \xi}, \xi\right)\right|^{2} \\
& \leqq\left(|T| U^{*} \xi, U^{* \xi}\right)(|T| \xi, \xi) \\
& =\left(U|T| U^{*} \xi, \xi\right)(|T| \xi, \xi) \\
& =\left(\left|T^{*}\right| \xi, \xi\right)(|T| \xi, \xi) .
\end{aligned}
$$

Hence, we conclude $\left|T^{*} i \leqq\right| T \mid$.

q.e.d.

From the above theorem, we easily see that the normality of $T$ implies (**). But, in general, the inequality (**) does not imply that $T$ is normal (for example, an isometry). However, when $T$ is compact, we obtain:

Theorem 1.2. Let $T \in \mathscr{B}(\mathscr{H})$ be compact. Then (**) holds for all $\xi, \eta \in \mathcal{H}$ if and only if $T$ is normal.

To prove Theorem 1.2 , we need the following fact due to T. Ando ( $[1] \cdots$ see also [4] and [9]):

Proposition 1.3. A compact hyponormal operator in $\mathcal{B}(\mathscr{H})$ is normal.

Proof of Theorem 1.2. Let $T=U|T|$ be the polar decomposition of a compact operator $T$. We must show that $\left|T^{*}\right| \leqq|T|$ implies the normality of $T$. By setting $S=U|T|^{1 / 2}$, we observe that

$$
\begin{aligned}
S S^{*} & =U|T| U^{*}=\left|T^{*}\right| \\
& \leqq|T|=|T|^{1 / 2} U^{*} U|T|^{1 / 2}=S^{*} S,
\end{aligned}
$$

i. e., $S$ is hyponormal. Since $T$ is compact, $S$ is also compact. Thus $S$ is actually normal by Proposition 1.3. On the other hand, since $S=U|T|^{1 / 2}$ is the polar decomposition of $S$, the normality of $S$ implies $U U^{*}=U^{*} U$ and $U|T|^{1 / 2}=$ $|T|^{1 / 2} U$. Thus, $U|T|=|T| U$, and hence $T$ is normal.

q. e. d

The function $t^{1 / 2}(t \geqq 0)$ is operator monotone $([7])$. Therefore, the hyponormality (i. e., $T T^{*} \leqq T^{*} T$ ) implies $\left|T^{*}\right| \leqq|T|$. But $\left|T^{*}\right| \leqq|T|$ does not necessarily imply the hyponormality of $T$. For example, consider the $2 \times 2$-matrices

$$
r=\left[\begin{array}{ll}
1 & 0 \\
0 & 0
\end{array}\right] \text { and } s=\left[\begin{array}{ll}
2 & 1 \\
1 & 1
\end{array}\right] .
$$

Note that $r \leqq s$ and $r^{2} \leqq s^{2}$. We set 


$$
T=\left[\begin{array}{llllll}
0 & & & & \\
r & 0 & & & \\
& s & 0 & & \\
& & s & 0 & \\
& & & \ddots & \ddots
\end{array}\right] .
$$

Then we compute

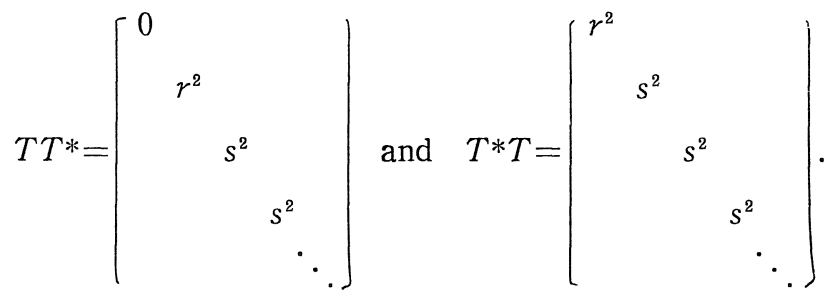

Therefore $\left|T^{*}\right| \leqq|T|$, but $T$ is not hyponormal (because of $r^{2} \$ s^{2}$ ).

\section{§2. Inequality $(*)$}

In this section, we consider the following inequality for an operator $T \in$ $\mathscr{B}(\mathscr{K})$ :

$$
|(T \xi, \xi)| \leqq(|T| \xi, \xi) \quad \text { for all } \xi \in \mathscr{H} .
$$

Obviously the inequality (**) implies (*), and hence the normality of $T$ implies (*).

For an operator $T \in \mathscr{B}(\mathscr{H}), \sup \{|(T \xi, \xi)|: \xi \in \mathscr{H},\|\xi\|=1\}$ is called the numerical radius of $T$ and denoted by $w(T)$. Then the following inequality is standard $([6])$ :

$$
(1 / 2)\|T\| \leqq w(T) \leqq\|T\|
$$

Theorem 2.1. For an operator $T \in \mathscr{B}(\mathscr{H}), \quad(*)$ holds for all $\xi \in \mathscr{H}$ if and only if

$$
U|T|^{1 / 2}=|T|^{1 / 2} X
$$

for some $X \in \mathscr{B}(\mathcal{H})$ with $w(X) \leqq 1$, where $T=U|T|$ is the polar decomposition of $T$.

Related results were obtained in [3].

Proof. Suppose that $(*)$ holds for all $\xi \in \mathscr{H}$. For each positive integer $n \in N$, we define $X_{n} \in \mathscr{B}(\mathscr{H})$ by

$$
X_{n}=\{|T|+(1 / n) I\}^{-1 / 2} U\{|T|+(1 / n) I\}^{1 / 2} .
$$

Then, for all $\xi \in \mathcal{H}$, 


$$
\begin{aligned}
\left(X_{n} \xi, \xi\right)= & \left(U\{|T|+(1 / n) I\}^{1 / 2} \xi,\{|T|+(1 / n) I\}^{-1 / 2} \xi\right) \\
= & \left(U\{|T|+(1 / n) I\}\{|T|+(1 / n) I\}^{-1 / 2} \xi,\{|T|+(1 / n) I\}^{-1 / 2} \xi\right) \\
= & \left(T\{|T|+(1 / n) I\}^{-1 / 2} \xi,\{|T|+(1 / n) I\}^{-1 / 2} \xi\right) \\
& +(1 / n)\left(U\{|T|+(1 / n) I\}^{-1 / 2} \xi,\{|T|+(1 / n) I\}^{-1 / 2} \xi\right) .
\end{aligned}
$$

Thus, by $(*)\left(X_{n} \xi, \xi\right)$ is majorized by

$$
\begin{aligned}
(|T| & \left.\{|T|+(1 / n) I\}^{-1 / 2} \xi,\{|T|+(1 / n) I\}^{-1 / 2} \xi\right) \\
& +(1 / n)\left\|\{|T|+(1 / n) I\}^{-1 / 2} \xi\right\|^{2} \\
= & \left(\{|T|+(1 / n) I\}\{|T|+(1 / n) I\}^{-1 / 2} \xi,\{|T|+(1 / n) I\}^{-1 / 2} \xi\right) \\
= & (\xi, \xi) .
\end{aligned}
$$

Therefore, we get

$$
w\left(X_{n}\right) \leqq 1 \text { and }\left\|X_{n}\right\| \leqq 2 .
$$

Thus, by the Alaoglu theorem, we can construct a subnet $\left\{X_{\jmath}\right\}_{\jmath \in J}$ converging weakly to some $X \in \mathscr{B}(\mathscr{H})$ with $\|X\| \leqq 2$ from the sequence $\left\{X_{n}\right\}_{n \in N}$. Then, we have $w(X) \leqq 1$ since

$$
(X \xi, \xi)=\lim _{j}(X, \xi, \xi) \leqq(\xi, \xi) .
$$

Now, from the definition of $\left\{X_{j}\right\}_{\jmath \in J}$, we get

$$
U\{! T \mid+(1 / F(j)) I\}^{1 / 2}=\{|T|+(1 / F(j)) I\}^{1 / 2} X_{F(\jmath)}
$$

for some mapping $F: J \rightarrow \boldsymbol{N}$ (in fact, $\left.X_{j}=X_{F^{(\jmath)}}\right)$. Hence, we conclude

$$
U|T|^{1 / 2}=|T|^{1 / 2} X
$$

by taking weak limits of both sides of (1) (see [7]).

Conversely, assume that $U|T|^{1 / 2}=|T|^{1 / 2} X$ for some $X \in \mathscr{B}(\mathscr{H})$ with $w(X)$ $\leqq 1$. Then, for all $\xi \in \mathscr{H}$,

$$
\begin{aligned}
\mid(T \xi, \xi) ! & =\left|\left(U|T|^{1 / 2}|T|^{1 / 2} \xi, \xi\right)\right| \\
& =\left|\left(|T|^{1 / 2} X|T|^{1 / 2} \xi, \xi\right)\right| \\
& =\left|\left(X|T|^{1 / 2} \xi,|T|^{1 / 2} \xi\right)\right| \\
& \leqq\left(|T|^{1 / 2} \xi,|T|^{1 / 2} \xi\right) \\
& =(|T| \xi, \xi) .
\end{aligned}
$$

From the above theorem, we easily obtain:

Corollary 2.2. When $T \in \mathscr{B}(\mathscr{H})$ satisfies $(*)$ for all $\xi \in \mathscr{K}$, we have 
and

$$
\left|T^{*}\right| \leqq 4|T|
$$

$$
U U^{*} \leqq U * U
$$

For matrices (i.e., $\operatorname{dim}(\mathscr{K})<\infty$ ), we obtain the following characterization:

Theorem 2.3. Let $\mathcal{H}$ be a finite-dimensional Hilbert space. Then for $T \in$ $\mathscr{B}(\mathscr{H}),(*)$ holds for all $\xi \in \mathscr{H}$ if and only if $T$ is normal.

Proof. We may assume that $\mathscr{H}=\boldsymbol{C}^{n}$. Then $\mathscr{B}(\mathscr{H})=M_{n}(\boldsymbol{C}) \quad(:=\{$ complex $n \times n$-matrix $\}$ ). Thanks to the obvious unitary invariance, we may and do assume that $T$ is of the form

$$
\left[\begin{array}{ccc}
a_{11} & \cdots & a_{1 n} \\
\ddots & \ddots & \vdots \\
& & a_{n n}
\end{array}\right]
$$

(i. e., $T$ is an upper triangular matrix).

We will show that

$$
a_{\imath \jmath}=0 \quad \text { if } i<j
$$

by induction on the size $n$ of a matrix.

For $n=1$ this result is trivial. Let

$$
T=\left[\begin{array}{cc}
a & \beta^{*} \\
0 & B
\end{array}\right] \text { and }|T|=\left[\begin{array}{cc}
x & \zeta^{*} \\
\zeta & Z
\end{array}\right] \text {. }
$$

Here, $a=a_{11}, x$ is a non-negative number, $\beta$ and $\zeta$ are (column) vectors in $\boldsymbol{C}^{n}$, $B$ is an upper triangular matrix in $M_{n}(\boldsymbol{C})$, and $Z$ is a positive matrix in $M_{n}(\boldsymbol{C})$. Then, since $T^{*} T=|T|^{2}$, we have

$$
|a|^{2}=x^{2}+\zeta * \zeta
$$

by comparing the $1-1$ components. Therefore $|a| \geqq x$. On the other hand, with $\xi={ }^{t}(1,0)$ in $C^{n+1}$, we have $|a| \leqq x$ by the assumption $(*)$. Hence

$$
x=|a| \text { and } \zeta=0 \text {. }
$$

Furthermore, since $T^{*} T=|T|^{2}$, we have

$$
\bar{a} \beta^{*}=0
$$

by comparing the $1-2$ components.

We will show $\beta=0$ by the contradiction argument. Then, the result follows from the induction hypothesis.

Assume $\beta \neq 0$, and hence $x=|a|=0$ from (2). We choose and fix a (column) vector $\xi^{\prime}(=\beta)$ in $C^{n}$ such that 


$$
k:=\beta^{*} \xi^{\prime}\left(=\beta^{*} \beta\right)>0 .
$$

Let $\xi={ }^{t}\left(p,{ }^{t} \xi^{\prime}\right)$ in $C^{n+1}(p>0)$. Since $a=0$ and $\zeta=0$, straight forward computations show

$$
(T \xi, \xi)=k p+\left(B \xi^{\prime}, \dot{\xi}^{\prime}\right)
$$

and

$$
(|T| \xi, \xi)=\left(Z \xi^{\prime}, \xi^{\prime}\right) \text {. }
$$

Therefore (*) does not hold for $p$ sufficiently large, a contradiction. q.e.d.

\section{§3. Relation of $(*)$ and $(* *)$}

From Theorem 1.2 and 2.3, for a finite-dimensional Hilbert space $\mathscr{H},(*)$ is equivalent to $(* *)$ (and to the normality of $T$ ). Recall that $(* *)$ implies $(*)$. But, in general, (*) does not imply (**) (i.e., $\left|T^{*}\right| \leqq|T|$ by Theorem 1.1).

We will consider an operator $T$ of the form

$$
T=\left[\begin{array}{cccc}
0 & & & \\
\alpha_{1} & 0 & & \\
& \alpha_{2} & 0 & \\
& \ddots & \ddots
\end{array}\right]
$$

to explain this phenomenon. Here, $\alpha_{n}$ 's are positive numbers to be fixed later. We note that

$$
|T *|=\left[\begin{array}{llll}
0 & & & \\
& \alpha_{1} & & \\
& & \alpha_{2} & \\
& & & \ddots
\end{array}\right] \text { and }|T|=\left[\begin{array}{llll}
\alpha_{1} & & & \\
& \alpha_{2} & & \\
& & \ddots & \\
& & & \ddots
\end{array}\right] .
$$

Therefore, if the sequence $\left\{\alpha_{n}\right\}$ is strictly decreasing, then $\left|T^{*}\right| \leqq|T|$ (i. e., $(* *)$ ) does not hold. On the other hand, Corollary 2.3 indicates that, if $\left\{\alpha_{n}\right\}$ decreases too rapidly, then $(*)$ does not hold either. Thus, we are forced to choose a slowly decreasing sequence $\left\{\alpha_{n}\right\}$ so that $T$ does not satisfy (**) but (*).

We set

$$
e_{n}^{-1}=3 \cdot 2^{n}-4 \quad(n \geqq 1) .
$$

By using this sequence $\left\{e_{n}\right\}$ (of positive numbers converging to 0 ), we set

$$
\alpha_{1}=1 \text { and } \alpha_{n+1}=\alpha_{n}\left(1+e_{n}\right)^{-1} \quad(n \geqq 1) .
$$

Then $\left\{\alpha_{n}\right\}$ is obviously decreasing, and it remains to show that (*) holds. For this purpose, we need the following result due to $\mathrm{T}$. Ando $([2]): w(Y) \leqq 1$ if and only if $Y=(I+A)^{1 / 2} B(I-A)^{1 / 2}$ with $-I \leqq A \leqq I$ and $\|B\| \leqq 1$ (we actually need just the easier half).

We define the sequence $\left\{a_{n}\right\} \subset[-1,1]$ (in fact, $a_{n} \in[-1,0)$ ) by

$$
a_{n}{ }^{-1}=-3 \cdot 2^{n-1}+2 \quad(n \geqq 1),
$$


and we set

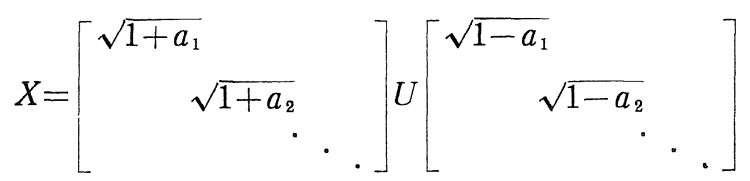

with

$$
U=\left[\begin{array}{ccccc}
0 & & & \\
1 & 0 & & \\
& 1 & 0 & \\
& & \ddots & \ddots
\end{array}\right]
$$

In fact, the above $U$ is the partial isometry appearing in the polar decomposition of $T$. Then, by T. Ando's result, we have $w(X) \leqq 1$. It is straightforward to see

$$
X=\left[\begin{array}{cccc}
0 & & & \\
\sqrt{1+e_{1}} & 0 & & \\
& \sqrt{1+e_{2}} & 0 & \\
& & \ddots & \ddots
\end{array}\right]
$$

thanks to

$$
1+e_{n}=\left(1+a_{n+1}\right)\left(1-a_{n}\right) .
$$

It is also easy to see

$$
U|T|^{1 / 2}=|T|^{1 / 2} X .
$$

Therefore, we see that $T$ satisfies $(*)$ by Theorem 2.1 .

Since $\sum_{n=1}^{\infty} e_{n}$ is convergent, so is

$$
\alpha_{1} \cdot \alpha_{n}{ }^{-1}=\prod_{i=1}^{n-1}\left(1+e_{i}\right)
$$

Therefore, $\lim _{n} \alpha_{n} ¥ 0$, and the above $T$ is not compact.

The author does not know whether the condition (*) and the normality are different for compact operators (in fact, we can confine ourselves to the case $T$ is compact quasi-nilpotent according to the way used in Theorem 2.3), and this problem seems to deserve further investigation.

\section{Appendix}

Theorem 2.3 is extended to trace class operators, that is, for them the condition $(*)$ is equivalent to the normality. We show this by the a method different from that of Theorem 2.3.

Lemma. Let $X \in \mathscr{B}(\mathscr{H})$ be a numerical contraction, i.e., $w(X) \leqq 1$. Then $X \xi=\xi$ implies $X^{*} \xi=\xi$. 
Proof. By T. Ando's factorization of a numerical contraction ([2]), there exist a self-adjoint contraction $A \in \mathscr{B}(\mathscr{H})$ and a contraction $B \in \mathscr{B}(\mathscr{H})$ such that

$$
X=(I+A)^{1 / 2} B(I-A)^{1 / 2}
$$

and $B$ is isometric on the range of $I-A$. Since

$$
\begin{aligned}
(\xi, \xi) & =(X \xi, \xi)=\left((I+A)^{1 / 2} B(I-A)^{1 / 2} \xi, \xi\right) \\
& \leqq\left\|B(I-A)^{1 / 2} \xi\right\| \cdot\left\|(I+A)^{1 / 2} \xi\right\| \\
& \leqq(1 / 2)\left\{\left\|(I-A)^{1 / 2} \xi\right\|^{2}+\left\|(I+A)^{1 / 2} \xi\right\|^{2}\right\} \\
& =(\xi, \xi),
\end{aligned}
$$

we have

$$
B(I-\Lambda)^{1 / 2} \xi=c(I+A)^{1 / 2} \xi
$$

for some scalar $c$ and

$$
((I-A) \dot{\xi}, \xi)=((I+A) \xi, \xi) .
$$

From (4), we have

$$
(A \xi, \xi)=0 .
$$

Since $B$ is isometric on the range of $(1-A)$, we have from (3)

$$
\begin{aligned}
(I-A) \xi & =(I-A)^{1 / 2} B^{*} B(I-A)^{1 / 2} \xi \\
& =c(I-A)^{1 / 2} B^{*}(I+A)^{1 / 2} \xi \\
& =c X^{*} \xi
\end{aligned}
$$

by the polarization identity. Therefore

$$
\begin{aligned}
(\xi, \xi) & =((I-A) \xi, \xi)=c\left(X^{*} \xi, \xi\right) \\
& =c(\xi, \xi)
\end{aligned}
$$

and hence $c=1$ and $X^{*} \xi=\xi-A \xi$. But since

$$
\begin{aligned}
\xi & =X \xi=(I+A)^{1 / 2} B(I-A)^{1 / 2} \xi \\
& =(I+A) \xi
\end{aligned}
$$

we obtain $X^{*} \xi=\xi$.

$$
\text { q. e. d. }
$$

Theorem. Let $T \in \mathscr{B}(\mathscr{K})$ be of trace class. Then (*) holds for all $\xi \in \mathscr{A}$ if and only if $T$ is normal.

Proof. By Theorem 2.1, there exists a numerical contraction $X \in \mathscr{B}(\mathscr{H})$ such that

$$
U|T|^{1 / 2}=|T|^{1 / 2} X
$$


where $T=U_{\mid} T \mid$ is the polar decomposition of $T$. The space $\mathcal{C}_{2}(\mathscr{H})$ of HilbertSchmidt class operators becomes a Hilbert space with the inner product $\langle K, L\rangle$ $=\operatorname{Tr}\left(L^{*} K\right)$ for $K, L \in \mathcal{C}_{2}(\mathcal{H})$. We define the operator $\Phi$ on $\mathcal{C}_{2}(\mathscr{H})$ by $\Phi(K)=$ $U^{*} K X$. Then $\Phi\left(|T|^{1 / 2}\right)=U^{* *}|T|^{1 / 2} X=|T|^{1 / 2}$.

Now, by T. Ando's factorization, we are led to the representation

$$
X=(I+A)^{1 / 2} B(I-A)^{1 / 2}
$$

with a self-adjoint contraction $A \in \mathscr{B}(\mathscr{H})$ and a contraction $B \in \mathscr{B}(\mathscr{H})$. Then

$$
\begin{aligned}
\Phi & =L_{U^{*}} R_{X} \\
& =L_{U^{*}} R_{(I-A) 1 / 2} \circ R_{B^{\circ}} R_{(I+A) 1 / 2} \\
& =R_{(I-A) 1 / 2} L_{U^{*}} R_{B^{\circ}} R_{(I+A) 1 / 2} \\
& =\left(I-R_{A}\right)^{1 / 2} \circ\left(L_{U^{*}} \circ R_{B}\right) \circ\left(I+R_{A}\right)^{1 / 2} .
\end{aligned}
$$

Here, $L_{D}$ and $R_{D}$ are the left- and right-multiplication operator on $\mathcal{C}_{2}(\mathscr{C})$ induced by $D \in \mathscr{B}(\mathscr{H})$ respectively. Again by T. Ando's result, we get $w(\Phi) \leqq 1$. By virtue of the above lemma, we have $\Phi^{*}\left(|T|^{1 / 2}\right)=U|T|^{1 / 2} X^{*}=|T|^{1 / 2}$ and hence

$$
U^{*}|T|^{1 / 2}=|T|^{1 / 2} X^{*} \text {. }
$$

Therefore, we get $\operatorname{Re}(U)|T|^{1 / 2}=|T|^{1 / 2} \operatorname{Re}(X)$ and $\operatorname{Im}(U)|T|^{1 / 2}=|T|^{1 / 2} \operatorname{Im}(X)$.

Let $\left\{E_{\operatorname{Re}(U)}(S): S\right.$ is a Borel subset of $\left.\boldsymbol{R}\right\}$ and $\left\{E_{\operatorname{Re}(X)}(S): S\right.$ is a Borel subset of $\boldsymbol{R}\}$ be the spectral projections of $\operatorname{Re}(U)$ and $\operatorname{Re}(X)$ respectively. Then, since $\operatorname{Re}(U)|T|^{1 / 2}=|T|^{1 / 2} \operatorname{Re}(X)$, we have $E_{\operatorname{Re}(U)}(S)|T|^{1 / 2}=|T|^{1 / 2} E_{\operatorname{Re}(X)}(S)$. This implies

$$
E_{\operatorname{Re}(U)}(S)|T| E_{\operatorname{Re}(U)}(S) \leqq|T| .
$$

But $E_{\operatorname{Re}(U)}(S)|T| E_{\operatorname{Re}(U)}(S) \leqq|T|$ is possible only when $E_{\operatorname{Re}(U)}(S)$ commutes with $|T|$. Therefore, we are led to the commutativity of $\operatorname{Re}(U)$ and $|T|$. In a similar fashion, $\operatorname{Im}(U)$ commutes with $|T|$. Hence, we obtain

$$
U|T|=|T| U,
$$

i. e., $T$ is quasi-normal. Since $T$ is of trace class, $T$ is normal by Proposition 1.3 . q. e d.

\section{References}

[1] Ando, T., On hyponormal operators, Proc. Amer. Math. Soc., 14 (1963), 290-291.

[2] - Structure of operators with numerical radius one, Acta Sci. Math. Szeged, 34 (1973), 11-15.

[3] - Structure of quadratic inequalities, J. Math. Anal. Appl., 70 (1979), $72-84$.

[4] Berberian, S.K., A note on hyponormal operators, Pacific J. Math., 12 (1962), 1171-1175.

[5] Conway, J.B. and Szymanski, W., Linear combinations of hyponormal operators, 
Rocky Mountain J. Math., 18 (1988), 695-705.

[6] Halmos, P.R., A Hilbert Space Problem Book, 2nd ed., Springer-Verlag, 1982.

[7] Reed, M. and Simon, B., Methods of Modern Mathematical Physics I: Functional Analysis, Academic Press, 1973.

[8] Saito, T., Hyponormal operators and related topics, Springer-Verlag, 247 (1972).

[9] Stampfli, J.G.. Hyponormal operators, Pacific J. Math., 12 (1962), 1453-1458. 
